\title{
Experimental technique of calibration of symmetrical air pollution models
}

\author{
P KUMAR \\ National Law University, Jodhpur, Rajasthan, India. \\ e-mail: pkumar_mbm@rediffmail.com
}

\begin{abstract}
Based on the inherent property of symmetry of air pollution models, a Symmetrical Air Pollution Model Index (SAPMI) has been developed to calibrate the accuracy of predictions made by such models, where the initial quantity of release at the source is not known. For exact prediction the value of SAPMI should be equal to 1 . If the predicted values are overestimating then SAPMI is $<1$ and if it is underestimating then SAPMI is $>1$. Specific design for the layout of receptors has been suggested as a requirement for the calibration experiments. SAPMI is applicable for all variations of symmetrical air pollution dispersion models.
\end{abstract}

\section{Introduction}

The transport and dispersion of suspended particulates in air, downwind of their point of discharge, is of great importance to statutory bodies, charged with the responsibility of ensuring that the process is in compliance with air pollution regulations. Most of the theoretical and numerical models are based on the basic premise of symmetry of dispersion of plumes on either side of the central line, downwind of the source. The pollutants may be gases or vapour or they may be particle matter of such a small size that their gravimetric settling may be neglected. In all these models the quantitative estimate of pollutants at any point away from the point of release, is normally given in fractions of the initially released total quantity at source $Q$. Hence predictions made by these models can be calibrated only when the quantity released from the source is correctly known. Due to instrumental errors or observational errors, the computed or observed value of $Q$ at source, may not be correct. This would result in incorrect predictions by the model. In this paper an attempt has been made to develop experimental technique to produce Symmetric Air Pollution Model Index (SAPMI) that could be used to test the accuracy of the predictions made through such models, before they are actually applied for regulatory purposes, without using the initially released total quantity $Q$.

\section{Principle of computing SAPMI}

It is generally assumed that unless horizontal wind shear persists, at or near the source of release of pollutants, the property of horizontal symmetry on either side of the central line (drawn downwind from the source) is maintained - whether it be plume or puff model for point, line or area sources. This concept could be used while developing the index SAPMI. This index is based on the experiment described in this paper, on the concept of dispersion-symmetry, by placing the receivers away from the source point, at and on either side of the central line of the plume, at equal lateral-distances from the line, as shown in figure 1.

Let the total quantity of the pollutant released from the source within finite time interval $=Q$ (unknown)

For the purpose of testing our model we assume total weight of dust initially released from source $=100$ units.

Keywords. Air pollution model; validation; SAPMI; predictions; receptors layout. 


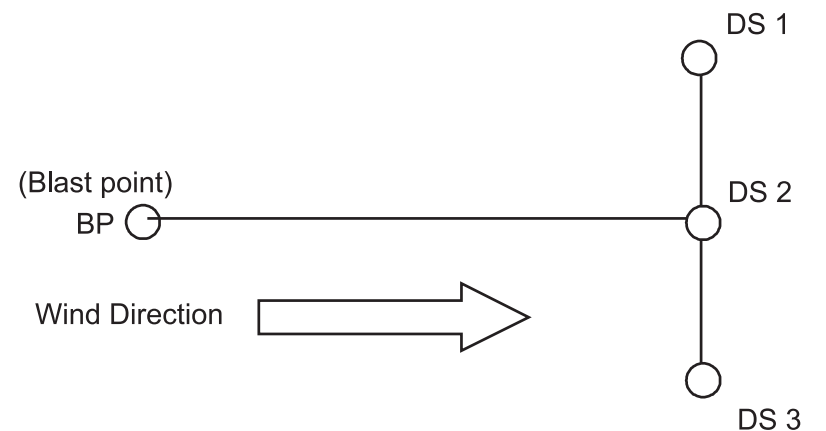

Figure 1. $\mathrm{BP}$ is the blast point. BP to DS-2 is the direction of the wind marked by arrow. Line DS-1 to DS-3 is at right angle to line joining $\mathrm{BP}$ and DS-2. Point DS-2 bisects the line joining DS-1 and DS-3. Distances from BP to DS-2, DS-2 to DS- 1 and DS-2 to DS-3 are $200 \mathrm{~m}, 50 \mathrm{~m}$ and $50 \mathrm{~m}$ respectively.

Based on this assumption let the predicted pollutant amount, by the model, at point DS-2 vide figure (1) be $P_{2}$.

But predicted dust amount will be different than actual amount of pollutant collected at point 2 since actual pollutant was $Q$ and not 100 units. Also let the quantity of actual measured pollutant at this point be $A_{2}$.

When $P_{2}$ is the predicted pollutant quantity at point DS-2 then the original total pollutant quantity at source is $=100$ units.

Hence when $A_{2}$ is the actual collected quantity of pollutant at point DS-2 then the total pollutant at original source

$$
=\left(100 / P_{2}\right) \times A_{2}=Q^{\prime} .
$$

But the original quantity of pollutant $(Q)$ will not be the same as obtained in equation (2) due to probable prediction-error $e_{M}$ in the model - if the model is incorrect.

Hence

$$
Q \neq Q^{\prime}=\left(100 / P_{2}\right) \times A_{2} .
$$

To make $Q^{\prime}$ equal to $Q$ equation (2) could be corrected with the prediction error $e_{M}$.

Hence after adding the correction $e_{M}$ with the predicted value $P_{2}$ we get

$$
Q=100 \times A_{2} /\left(P_{2}+e_{M}\right) .
$$

Total quantity $Q$ computed by (4) can be used to predict the quantity of pollutant at site DS-1 and site DS-3 as $\left\{\left(P_{1} \times A_{2}\right) /\left(P_{2}+e_{M}\right)\right\}$ and $\left\{\left(P_{3} \times\right.\right.$ $\left.\left.A_{2}\right) /\left(P_{2}+e_{M}\right)\right\}$ respectively. Symmetry in dispersion process as per the model however provides that error in the measurement and the prediction at site 1 and 3 should also be equal.
Hence

$$
\begin{aligned}
A_{1} & -\left\{\left(P_{1} \times A_{2}\right) /\left(P_{2}+e_{M}\right)\right\} \\
= & A_{3}-\left\{\left(P_{3} \times A_{2}\right) /\left(P_{2}+e_{M}\right)\right\} .
\end{aligned}
$$

Solving equation (5) for $e_{M}$ we get

$$
e_{M}=\left\{\left(P_{1} \times A_{2}-P_{3} \times A_{2}\right) /\left(A_{1}-A_{3}\right)\right\}-P_{2} .
$$

Equation (6) gives the quantity of error in the model. Hence for the condition of no error, i.e., for $e_{M}=0$, equating (6) to zero we obtain the condition for accuracy of the model, i.e.,

$$
\left(A_{1}-A_{3}\right) / A_{2}=\left(P_{1}-P_{3}\right) / P_{2}
$$

or

$$
\frac{\left(A_{1}-A_{3}\right) / A_{2}}{\left(P_{1}-P_{3}\right) / P_{2}}=1=\text { SAPMI } .
$$

Equation (7) gives the condition of the accuracy of the prediction made by any model. Thus if,

$$
\begin{aligned}
\text { SAPMI } & =1 \text { (prediction is accurate) } \\
& <1 \text { (model is overestimating) } \\
& >1 \text { (model is underestimating). }
\end{aligned}
$$

\section{Experiment for calibration}

Calibration experiments were performed at Birla cement factory, Chanderia, which is located in the southern region of the state of Rajasthan, India. Limestone quarrying is done by blasting the ground crust with detonators. This loosens the crust for mineral collection but it spews sufficient dust in atmosphere.

\subsection{Measurement of dust}

High volume air samplers are used for the estimation of the mass $(M)$ of the SPM in a given volume of air $(S \cdot \tau)$, where $S$ is the known rate at which air is drawn through the apparatus and $\tau$ is the duration of the sampling process. If $\chi$ (mass of dust per unit volume) is the instantaneous concentration at the sampling position, the average concentration over the duration of sampling is

$$
\bar{\chi}=\frac{1}{\tau} \int_{0}^{\tau} \chi \mathrm{d} t=\frac{M}{S \tau} .
$$

At the quarry site three Respirable Dust Samplers (RDS) were installed as per layout design shown in figure 1. Measurements of respirable dust 
$\left(\mathrm{PM}_{10}\right)$ were taken on Whatman filter papers. Papers were weighed before and after the collection of dust in RDS. Papers were changed at the interval of 15 minutes. All the samplers were simultaneously started/shut-off by a single switch point. 20 minutes before the blast, first paper was installed and 15 minutes later second paper was replaced. Thus each paper collected dust for 15 minutes from 18 litre of ambient air, at the rate of 1.2 litre per minute. As the RDS were started 20 minutes before the blast hence after 15 minutes of running of machine (i.e., 5 minutes before the blast) filtered dust on each Whatman-paper gave the background dust in the ambient atmosphere. Immediately, therefore, second set of filter papers were replaced in all the three machines. Mine-blast was made 5 minutes later and 10 minutes, thereafter, third set of papers were again replaced. After this 4th and 5th set of papers were replaced at the intervals of 15 minutes each. Each unused filter paper was dried and weighed before use. Total dust accumulated at the filter paper was determined by subtracting the weight of unused filter paper from that of used filter paper. Dust per litre of air at $1 \mathrm{~m}$ a.g.l. at known distances (refer figure 1), from the point of blast was determined by dividing the dust weight on each filter paper by ' 18.0 '.

\subsection{Safety measures}

During the blasts stones are thrown out hence to avoid damage to RDS instruments and personnel, instruments at location DS-2 was kept at $200 \mathrm{~m}$ away from the point of blast and distances between DS-2 to DS-3, and DS-2 to DS-1 were kept as $50 \mathrm{~m}$ each.

\subsection{Measurements of surface wind}

The quantity $(M)$ in equation (8) is a function of displacement in wind direction (along $\mathrm{x}$-axis), cross-wind displacement (along y-axis) and vertical displacement (along z-axis) from central point of dust cloud for the modelling of the dispersion process explicit relationship between cross wind spread and the statistics of wind fluctuation is needed. Cross wind dimension of the dispersing dust cloud is conventionally represented either by width $\left(Y_{0}\right)$ between position at which concentration falls $1 / 10$ th of the central peak value or by standard deviation $(\sigma)$ of cross-wind displacement of the material which in practice is defined by

$$
\sigma_{y}^{2}=Y^{2}=\frac{\Sigma Y^{2}}{\Sigma M}-\left[\frac{\Sigma M Y}{\Sigma M}\right] .
$$

Either definitions are related since $Y_{0}=2.15 \sigma$. It is noted that if standard deviation of wind direction

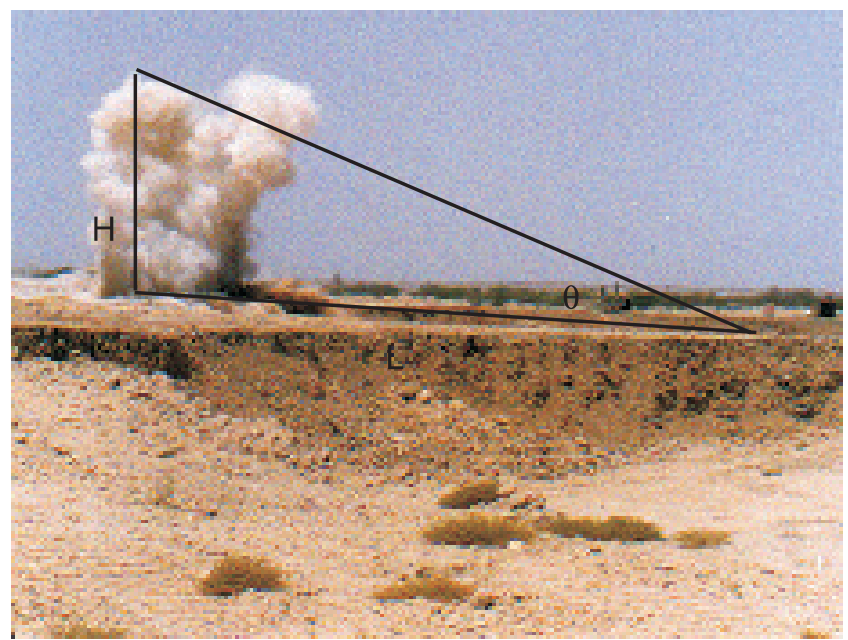

Figure 2. Measurement of the height of the blast column with the help of horizontal distance $(L)$ and angle $(\theta)$ of the inclination of top of dust-cloud mushroom.

is $\sigma_{\theta}$ and speed is $\sigma_{s}$ then $\sigma /\left(\sigma_{\theta} x\right)$ or $\sigma /\left(\sigma_{s} t\right)$ do closely approach to unity on an average for time $(t)$ and distance $(x)$. Hence, good correlation between dust cloud spread and the intensity of the turbulence exists irrespective of the condition of the surface reference and stability. This is a significant result, in the theoretical modelling of the dispersing puff of dust cloud. Hence an anemometer was installed close to blast site and wind measurement was made and $\sigma_{s}$ values were computed.

\section{Blast mushroom}

Figure 2 shows the mushroom of dust cloud immediately after the blast. $L=$ Horizontal distance of the theodolite. With the help of theodolite angle ' $\theta$ ' of the top of the dust cloud was determined.

Height of the dust column is given by $H=$ $L \tan (\theta)$.

\section{Modelling of dispersion of dust mushroom}

The most recent theory of dispersion modelling is known as gradient transfer theory (or K-theory) which employs the basic differential equation that attempts to describe the advective and turbulent motion of the atmosphere. The other one is a statistical theory. With increasingly better understanding of the convective boundary layer's (CBL) parameterization process, several numerical and analytical solutions have also been developed for dispersion. Gaussian plume diffusion model, which is based on the simple statistical theory, assumes normal distribution as representing dispersion of 
gas and SPM in atmosphere. This model, although, raises several questions with respect to its assumptions of continuous source geometry, slender plume, neglect of longitudinal diffusion in comparison to advective term by mean flow, but due to its simple form it constitutes the basis of many atmospheric dispersion calculations for regulatory applications (Taylor 1921, 1959; Batchelor 1949; Csanady 1973; Beryland 1975). For completeness, gradient transfer theory and statistical theory are both discussed briefly hereunder.

\subsection{Gradient transfer theory}

For any perturbation parameter $\xi^{\prime}$ multiplied by the motion term the gradient transport theory assumes

$$
\overline{u^{\prime} \xi^{\prime}}=-K \frac{\overline{\partial \xi}}{\partial x}
$$

where $K$ implies $\overline{u^{\prime} \xi^{\prime}}$ flows down the local gradient of $\xi^{\prime}$. Although it is the simplest parameterization, known on first order closer relationship, it often fails when larger eddies are present in the flow, as found in convective boundary layer (CBL). Hence its validity is useful for near-neutral or stable atmospheric conditions, only. Batchelor (1949) and Csanady (1973), have shown that for the solution of diffusion equation to be consistent with the result of the statistical theory of diffusion, the apparent eddy diffusivities must be related to the plume dispersion parameters as

$$
K_{x}=\frac{\bar{u}}{2} \frac{\partial \sigma_{x}^{2}}{\partial x}, \quad K_{y}=\frac{\bar{u}}{2} \frac{\partial \sigma_{y}^{2}}{\partial x}, \quad K_{z}=\bar{u} \frac{\bar{u}}{2} \frac{\partial \sigma_{z}^{2}}{\partial x} .
$$

If $T_{L u}$ is the integral time scale of Lagrangian velocity fluctuations in the $x$-direction and $L_{x}=u T_{L u}$, then

$$
\begin{aligned}
& K_{x}=\sigma_{u}^{2} t=\frac{\sigma_{u}^{2}}{\bar{u}}, \quad \text { for } x \leq L_{x}, \\
& K_{x}=\sigma_{u}^{2} T_{L u}=\frac{\sigma_{u}^{2} L_{x}}{\bar{u}}, \quad \text { for } x \geq L_{x} .
\end{aligned}
$$

Alternatively following general forms can be used

$$
\begin{aligned}
& K_{x}=\frac{\sigma_{u}^{2} x}{\bar{u}}\left[1+\frac{x}{c L_{x}}\right]^{-1}, \\
& K_{y}=\frac{\sigma_{u}^{2} x}{\bar{u}}\left[1+\frac{x}{c L_{y}}\right]^{-1},
\end{aligned}
$$

for all ranges, in which $c$ is a constant of the order of 1 .

\subsection{Statistical theory}

With its inherent assumptions it is strictly valid only in the region of uniform flow and homogeneous turbulence and is most suitable for convective boundary layer (CBL). For highly convective light wind conditions, plume dispersion parameter should be specified on the basis of the mixed layer similarity theory (Arya 1995) as below:

$$
\sigma_{*}=\frac{a t_{*}}{\left(1+h t_{*}\right)^{1 / 2}} .
$$

Here

$$
\sigma_{*}=\frac{\sigma_{x}}{h}=\frac{\sigma_{y}}{h}=\frac{\sigma_{z}}{h} \quad \text { and } \quad t_{*}=\frac{t w_{*}}{h},
$$

where $h$ is the height of convective boundary layer (CBL).

\section{Model I}

As the order of the dispersion range $(O \approx 100)$ is higher than the order of the thickness of the dustmushroom $(O \approx 10)$, hence neglecting thickness of the dust-mushroom, one can obtain the cumulative dispersion effect by integrating effects of all the elevated source elements from surface to height $H$ i.e., top of the dust cloud. Near-field concentration from an instantaneous blast mushroom source for flat surface (Kumar and Bhandari 2000), therefore, could be computed through the following integral equation:

If $H_{*}=H / h$

$$
\begin{aligned}
& \bar{C}\left(x_{*}, y_{*}, z_{*}, t_{*}\right)=\frac{1}{(2 \lambda)^{3 / 2}} \int_{0}^{H_{*}} \frac{1}{\sigma_{*}^{3}} \exp \left[\frac{\left(x_{*}-\overline{u_{*} t_{*}}\right)^{2}}{2 \sigma_{*}^{2}}\right] \\
& \quad \times \exp \left[\frac{y_{*}^{2}}{2 \sigma_{*}^{2}}\right] \exp \left[\frac{\left(z_{*}-H_{*}^{\prime}\right)^{2}}{2 \sigma_{*}^{2}}\right] \\
& \quad+\exp \left[\frac{\left(z_{*}-H_{*}^{\prime}\right)^{2}}{2 \sigma_{*}^{2}}\right] d H_{*}^{\prime} .
\end{aligned}
$$

Ground level concentration (GLC) at the plume centerline $\left(y_{*}=0 ; z_{*}=0\right)$ could be obtained from

$$
\begin{aligned}
\bar{C}\left(x_{*}, t_{*}\right)= & \frac{1}{(2 \pi)^{3 / 2}} \int_{0}^{H_{*}} \frac{2}{\sigma_{*}^{3}} \exp \left[\frac{\left(x_{*}-\overline{u_{*} t_{*}}\right)^{2}}{2 \sigma_{*}^{2}}\right] \\
& \times \exp \left[\frac{H_{*}^{\prime 2}}{2 \sigma_{*}^{2}}\right] d H^{\prime} .
\end{aligned}
$$




\section{Model II}

Model I was improved with the introduction of actual surface profile as the lower boundary condition. All the heights are taken from the blast point as a reference level. The $\mathrm{x}$-axis is taken in the mean wind. $y$-axis is at lateral direction at right angle to x-axis. z-axis is vertical distance from the location of blast. Ground simulation is achieved through MATLAB package which is based on leastsquare approximation. It was achieved in two steps; preliminary best fit at the first step was linearly added with the best fit of the errors in the second step. Preliminary best-fit sixth order polynomial, i.e.,

$$
P_{p}(x)=\sum_{i=0}^{6} a_{i} x^{i} .
$$

It has been obtained for the ground profile below the central line of dispersed plume. Following a similar approach, to achieve a better fit to the terrain another correction-polynomial,

$$
P_{c}(x)=\sum_{i=0}^{6} b_{i} x^{i}
$$

was obtained for departure $\left(P_{p}-Z_{a}\right)$ from the actual $\left(Z_{a}\right)$ contour heights. Final best fit $\left(Z_{t}\right)$ was computed by linear addition of preliminary polynomial $P_{p}(x)$ (equation 20) with the correction polynomial $P_{c}(x)$ (equation 21 ) as,

$$
Z_{t}=P_{p}(x)+P_{c}(x)=\sum_{i=0}^{6}\left(a_{i}+b_{i}\right)_{i} x^{i} .
$$

If the variation in the topography in height is less than the vertical height of the dust mushroom then reflection theory approach could still be assumed to be valid to a reasonable degree of accuracy. Equations (18) and (19) would, therefore, still remain valid.

\section{Model III}

Model II was better than model I as it included the ground profile, too, as a lower boundary condition. But since the vertical height of the dust mushroom in surface mine blast goes up to 30 to 50 meters high hence a more appropriate modelling would be with the inclusion of vertical wind shear. Model II was, therefore, further improvised by inclusion of power law in the observed surface winds, by the in situ anemometer.

\subsection{Power law}

Power law for the vertical variation of mean wind speed is given by

$$
\frac{u_{2}}{u_{1}}=\left[\frac{z_{2}}{z_{1}}\right]^{\alpha},
$$

where $u_{1}$ and $u_{2}$ are the mean wind speed at heights $z_{1}$ and $z_{2}$ respectively and $\alpha$ is power law index as under,

$$
\alpha=\frac{\log u_{2}-\log u_{1}}{\log z_{2}-\log z_{1}} .
$$

Wind velocity $u_{2}$, at different levels of the sourceelements of dust mushroom, could be accordingly determined if value of $u_{1}$ (i.e., observed surface wind) and height of the source-element above ground level and value of $\alpha$ are known. Power law factor $(\alpha)$ for different stations of India has been documented in Appendix "A" (Anna Mani 1995). $\alpha$ value for Birla Cement Factory, Chittorgarh, for the month of December was obtained as equal to 0.15 . This value was included in model III.

\subsection{Correction due to terrain forcing factor}

Terrain response to dispersion differs with the type of stability conditions in the atmosphere. Empirical relation which gives terrain forcing factor $\left(F_{T}\right)$ is mentioned below.

$$
F_{T}=\left(H_{A}-H+\Delta E\right) / \Delta E
$$

or

$$
H_{A}=H-\Delta E+F_{T} \Delta E
$$

where

$$
\begin{aligned}
H_{A} & =\text { Adjusted effective height } \\
H & =\text { Effective height } \\
\Delta E & =E_{R}-E_{S} \\
E_{R} & =\text { Ground level elevation of receptors } \\
E_{S} & =\text { Ground level elevation of source } \\
F_{T} & =0 \text { for stable } \\
F_{T} & =1 \text { for unstable } \\
F_{T} & =0.1 \text { for neutral stability. }
\end{aligned}
$$

Figure 3 shows vertical dispersion over undulated terrain under unstable, stable and neutral stability conditions.

As has been explained in para 6, above, vertical dust column is assumed to be formed of small dust 


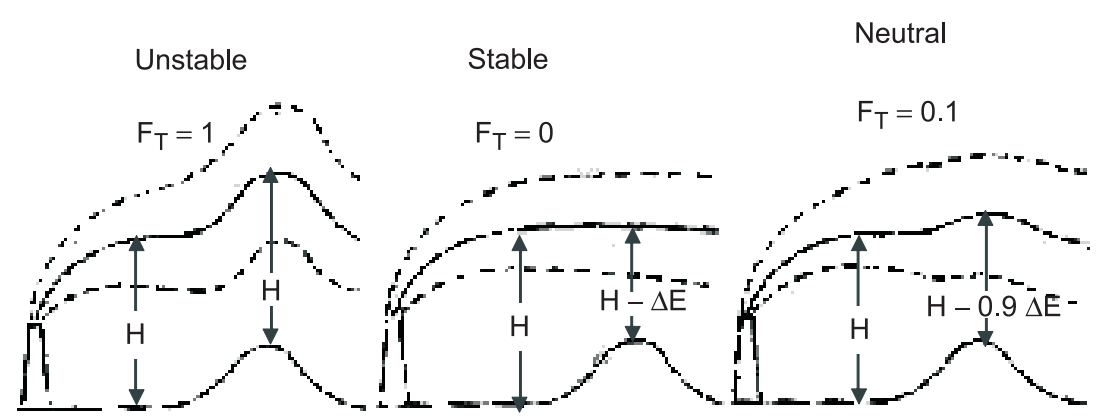

Figure 3. Adjusted effective height $\left(H_{A}\right)$ of the central line of dispersed plume at various distances from the elevated source for unstable $\left(F_{T}=1\right)$, stable $\left(F_{T}=0\right)$ and neutral $\left(F_{T}=0.1\right)$ atmospheric conditions.

Table 1. .

\begin{tabular}{ll}
\hline \multicolumn{1}{c}{ Description } & Assumed value \\
\hline Distance in meter from blast site & $100.00 \mathrm{~m}$ \\
Angle of the top of the mushroom from & $45^{\circ}$ \\
$\quad$ the point of observation & $980 \mathrm{hPa}$ \\
Surface pressure in $\mathrm{hPa}$ & $5 \mathrm{~m} / \mathrm{s}$ \\
Surface wind speed & $0.005 \mathrm{~m} / \mathrm{s}$ \\
Wind variation from mean wind, speed & $8 \mathrm{~m} / \mathrm{s}$ \\
Wind speed at $850 \mathrm{hPa}$ & $5 \mathrm{~g} / \mathrm{k}$ \\
Saturation mixing ratio at surface & $2 \mathrm{~g} / \mathrm{k}$ \\
Saturation mixing ratio at $850 \mathrm{hPa}$ & 1200 \\
Mixing condensation level & $25^{\circ}$ \\
Latitude of blast site & 596 \\
Height (above sea level) of blast point & $1500 \mathrm{~m}$ \\
\hline
\end{tabular}

source elements stacked-up together. Each element of dust can be assumed as the elevated point source similar to chimney-source, shown in figure 3. The dispersion results are based on a horizontally moving frame of reference; hence as the frame moves along the topography necessary correction in the effective height to the source element has to be applied. In model III, therefore, $F_{T}$ - correction for dispersion in vertical, has also been applied.

\section{Data}

Preliminary input-data were used for the computations presented in table 1 .

\section{Discussion}

All the three models were used to study the SAPMI values. Table 2 presents the results obtained by different models. Gradual improvement in the value of SAPMI was noticed from model I to model III.
Table 2.

\begin{tabular}{cc}
$\begin{array}{c}\text { Type of } \\
\text { model }\end{array}$ & Computed SAPMI values \\
\hline Model I & SAPMI $=\frac{\left(A_{2}-A_{4}\right) / A_{3}}{\left(P_{2}-P_{4}\right) / P_{3}}=\frac{5.20 \times 10^{-3}}{1.33 \times 10^{-3}}=3.9$ \\
Model II & SAPMI $=\frac{\left(A_{2}-A_{4}\right) / A_{3}}{\left(P_{2}-P_{4}\right) / P_{3}}=\frac{5.20 \times 10^{-3}}{2.17 \times 10^{-3}}=2.39$ \\
Model III & SAPMI $=\frac{\left(A_{2}-A_{4}\right) / A_{3}}{\left(P_{2}-P_{4}\right) / P_{3}}=\frac{5.20 \times 10^{-3}}{3.93 \times 10^{-3}}=1.32$ \\
\hline
\end{tabular}

This indicated that as the error $e_{M}$ in the model predictions were reduced by applying better physical parameterizations vis-à-vis increasing the prediction accuracy, SAPMI values approached more and more near 1 .

SAPMI values decreased from 3.9 to 2.39 in model II as the undulated topography was incorporated in model I. More perfection in the model, however, was achieved in model III with the introduction of topography, ground response and vertical wind shear. This brought down the SAPMI value from 2.39 to 1.32. Still, we observe, that its value is not exactly unity. This may be due to inherent limitations in the assumptions that vertical dust cloud has uniform distribution of dustdensity and many experimental or observational errors which affect the numerator of the SAPMI computation values. They could be as under:

- Incorrect identification of central line of plume.

- Different rate of suction by different dustsamplers due to variation in the manometer readings in the field.

- Assumption of constant wind with height.

- Difference in elevation of dust samplers kept at sites 1, 2 and 3 due to variation in ground contour in the field.

- Fugitive loose dust (sucked in the instrument) may affect the accuracy. Further such a fugitive dust error may not be uniform for all the sites. 
Beside experimental causes there could be mathematical approximation errors, too, as follows:

- Neglect of thickness of the vertical dust column.

- Application of reflection property with the assumption that the height of the blast column is of a higher order than the order of variation in the ground contour elevation.

Nevertheless it may be clearly observed that SAPMI shows steady approach to unity as the errors of the models are increasingly diluted with better parameterizations, step by step. In other words higher is the value of SAPMI's departure from unity, i.e., the higher the value of (SAPMI 1 ), the higher is the range of error in the predicted values through the models.

\section{Conclusions}

- Symmetrical Air Pollution Model Index

$$
\mathrm{SAPMI}=\left\{\left(\left(A_{2}-A_{4}\right) / A_{3}\right) /\left(\left(P_{2}-P_{4}\right) / P_{3}\right)\right\}
$$

is a useful parameter to test the accuracy of predictions made by the models where the initial mass of vertical column is unknown.

- With gradual improvement in the physics of the model SAPMI value gradually approaches to unity.

- Once the extent of model accuracy is established, its inaccuracy range could be accordingly accounted while interpreting the results of the predictions.

- Even if at the application stage of the model, the initial quantity of pollutants released at the source point is accurately known, the predictions with the model would not be correct unless SAPMI value is equal to one.

\section{Acknowledgements}

Author acknowledges with thanks the efforts of Shri Anoop Banerjee, Shri Vikas Mohan, senior geologist, in helping to conduct the field experiments at M/s. Birla Cement Works, Chittorgarh, Rajasthan, India. Author is also indebted to Ms. Urvashi Shrivastava and Shri Anoop Banerjee in preparing and typing the manuscript. Author is also extremely grateful to $\mathrm{M} / \mathrm{s}$. Birla Cement Works, Chittorgarh, Rajasthan, India, for extending all the facilities and helping hands for the experiments.

\section{References}

Anna Mani 1995 Wind Energy (Resource Survey in India); (Allied Publishers Ltd., New Delhi).

Arya S P 1995 Modelling and Parametarization of NearSource diffusion in weak winds; J. Appl. Met 34 $1112-1122$.

Batchelor G K 1949 Diffusion in a field of homogeneous turbulence, I. Eulerian analysis; Aust J. Sci. Res. 2 437-450.

Berlyand M E 1975 Contemporary Problems of Atmospheric Diffusion and Air Pollution; (Hydromet. Press), p. 448.

Csanady G T 1973 Turbulent Diffusion in the Environment; (D. Reidel Publishing) p. 248.

Kumar P and Bhandari S 2001 Modelling of Near Source Dust Dispersal after the opencast mine Blasting on flat surface in weak wind in Tropical Countries; Proceedings of EXPLO-2001, Hunter Valley, NSW, Australia, 28th31st October 2001, p. 289-293.

Taylor G I 1921 Diffusion by continuous movements; Proc. London Math. Soc. 2 196-212.

Taylor G I 1959 The present position in the theory of turbulent diffusion; Adv. Geophys. 6 101-111. 